\title{
Zinner's syndrome, radiological diagnosis for a rare syndrome with non-specific clinical presentation: case report
}

\author{
Ahmed Atef Soliman* ${ }^{*}$, Anwar Ali Alshukami and Walaa Hussein AlZuber
}

\begin{abstract}
Background: Zinner's syndrome is a rare congenital malformation involving the seminal vesicle that mostly shows cystic transformation. Embryogenesis of the kidney, ureter, seminal vesicle, and vas deferens can be altered if an insult occurs during the first trimester mainly between the 4th and the 13th gestational week. Cystic lesions of the seminal vesicle may present with a mass effect. Associated ipsilateral renal agenesis is a characteristic feature in more than $50 \%$ of patients as the seminal vesicles and ureteral buds originate from the mesonephric (Wolffian) duct. For management, the transrectal ultrasound provides good visualization of the seminal vesicles and the rest of the pelvic structures providing a good guidance for aspiration of the cysts.
\end{abstract}

Case presentation: We present a case of a seminal vesicle fluid-filled tubular lesion. The patient first complained of vague abdominal pain with lower abdominal fullness. He was clinically evaluated, and radiological evaluation was performed using different modalities, and finally, the patient took his decision to live with it without any intervention at present.

Conclusions: The combination of seminal vesicle cysts and ipsilateral renal agenesis, is a rare urological anomaly with an insult during the 1st trimester is to be considered as the first suspicion. Usual manifestations are caused by the seminal vesicle cysts that cause mass effect and irritation to the surroundings in the form of urinary bladder irritation and in sometimes obstruction as well as pain/discomfort in the perineum and scrotum. Epididymitis is frequently occurring as a complication. Treatment mainly consists of removing the seminal vesicle cyst/tubular lesion.

Keywords: Zinner's syndrome case report, Seminal vesicle cystic lesion, Unilateral renal agenesis, Ejaculatory duct obstruction

\section{Background}

Zinner's syndrome is a rare urogenital congenital developmental anomaly that occurs in the early embryogenesis affecting the distal portion of the Mullerian duct. Patients with Zinner's syndrome mainly show triad of unilateral renal agenesis, ipsilateral seminal vesicle obstruction with cystic transformation, and ipsilateral ejaculatory duct obstruction. Zinner described this

* Correspondence: dr.soliman.ahmed.82@gmail.com

Radiology Department, King Khalid Hospital, Najran 66262, Saudi Arabia syndrome for the first time in 1914, and about 200 cases have been reported in the literature [1].

The syndrome usually presents in the second and third decade of life mainly (after the beginning of sexual activity) and usually in the form of voiding manifestations such as dysuria, prostatism, urgency, painful ejaculation, hematospermia, perineal pain/discomfort, and sometimes infertility [2].

In this paper, we will present a case of a 35-year-old patient who presented with vague abdominal pain and pelvic fullness. A brief review of the literature was considered regarding the developmental anomaly details,

\section{Springer Open}

(c) The Author(s). 2020 Open Access This article is licensed under a Creative Commons Attribution 4.0 International License, which permits use, sharing, adaptation, distribution and reproduction in any medium or format, as long as you give appropriate credit to the original author(s) and the source, provide a link to the Creative Commons licence, and indicate if changes were made. The images or other third party material in this article are included in the article's Creative Commons licence, unless indicated otherwise in a credit line to the material. If material is not included in the article's Creative Commons licence and your intended use is not permitted by statutory regulation or exceeds the permitted use, you will need to obtain permission directly from the copyright holder. To view a copy of this licence, visit http://creativecommons.org/licenses/by/4.0/. 
main clinical presentations, image findings, and possible management pathways.

\section{Case presentation}

A 35-year-old male patient presented to the outpatient clinics with vague abdominal pain and pelvic fullness, there were no urinary manifestations, and the patient is married with 4 children. On examination, there was discomfort with palpation of the pelvis. After reviewing the patient, the responsible physician requested a full abdominal and pelvic US, urine analysis, PSA, and $\mathrm{CBC}$ for evaluation.

Urine analysis, PSA, and CBC came out normal with no signs of urinary tract infection or crystals to suggest urinary tract stones.

Trans-abdominal US study showed a fluid-filled cystic lesion involving mainly the region of the right seminal vesicle measuring about $6 \times 6 \times 8 \mathrm{~cm}$ in its maximum antro-posterior, transverse, and craniocaudal dimensions, respectively; the lesion is inseparable from the right upper surface of the prostate. The right kidney was not visualized with mild compensatory hypertrophy of the left kidney. In the setting of absent history of previous surgical intervention, our team considered the more frequent possibilities as ectopic pelvic right kidney with long-standing pelviureteric junction obstruction; however, the possibility of cystic malformation of the right seminal vesicle was mentioned later on as a rare possibility (Fig. 1).

The patient then had a CT pyelogram that confirmed the absence of the right kidney with no renal artery or vein seen while the pelvic lesion was seen of low attenuation with no enhancement at any phase exerting mild mass effect upon the surrounding structures, the rest of the findings were as seen in the US including the mild compensatory hypertrophy of the left kidney, and the rest of the solid abdominal organs and bowel appeared unremarkable (Fig. 2).

Pre- and post-IV contrast MRI of the pelvis was done 1 week later that showed tubular fluid-filled structure (high signal in T2 WIs and of slightly high signal in the T1 images 2ry to its high protein contents) that is replacing the right seminal vesicle with no enhancement in the post-contrast images; it overall measured about $6.9 \times 6.5 \times 8.6 \mathrm{~cm}$ in its maximum cross-sectional and CC dimensions, respectively; otherwise, the left seminal

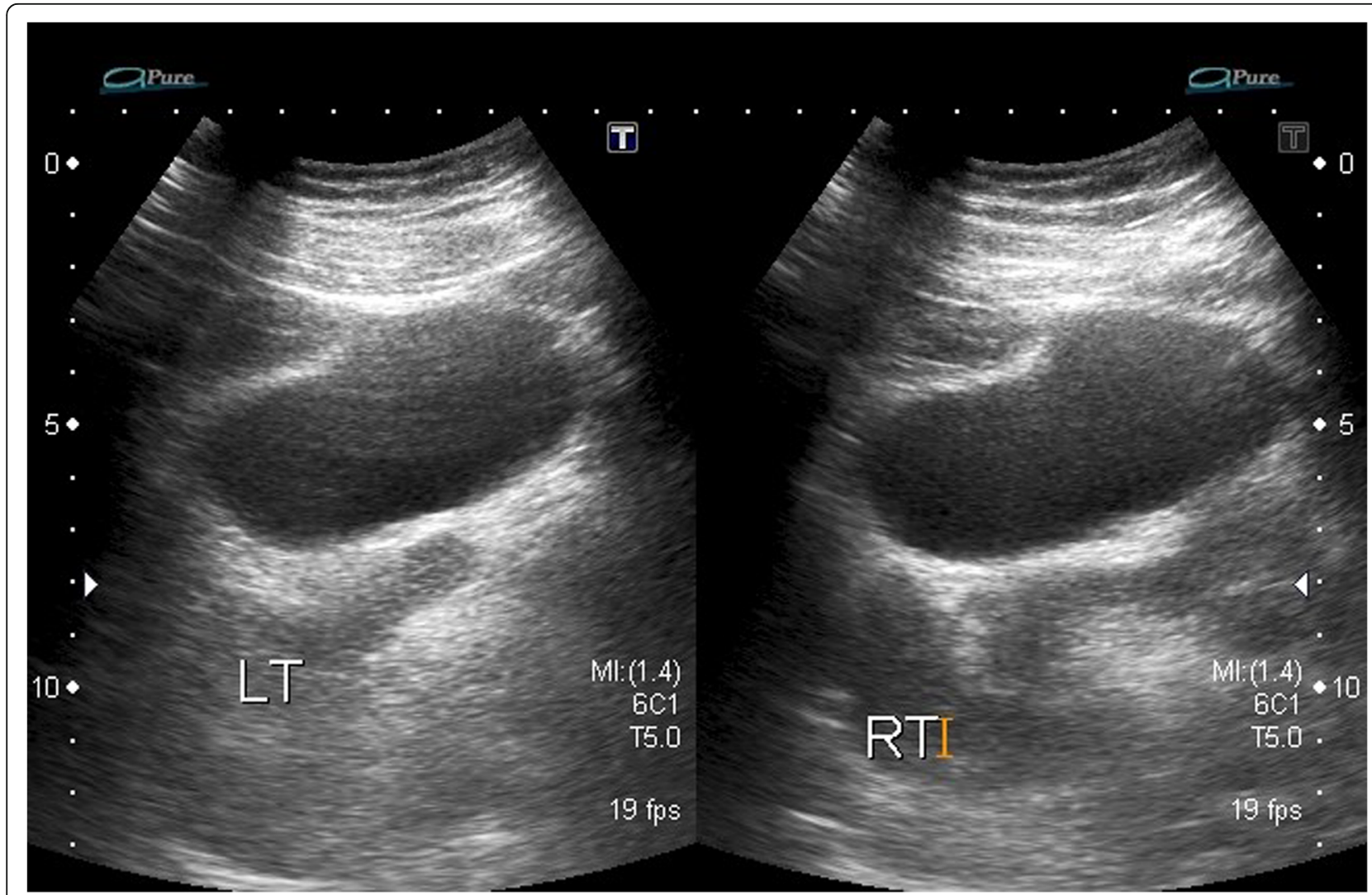

Fig. 1 US images of the pelvis showing a lobulated cystic lesion with rather clear contents and posterior enhancement arising from the right seminal vesicle measuring about $6 \times 6 \times 8 \mathrm{~cm}$ in its maximum dimensions and indenting the UB and prostate; otherwise, the normal sonographic appearance of the other pelvic structures including the left vesicle is preserved 


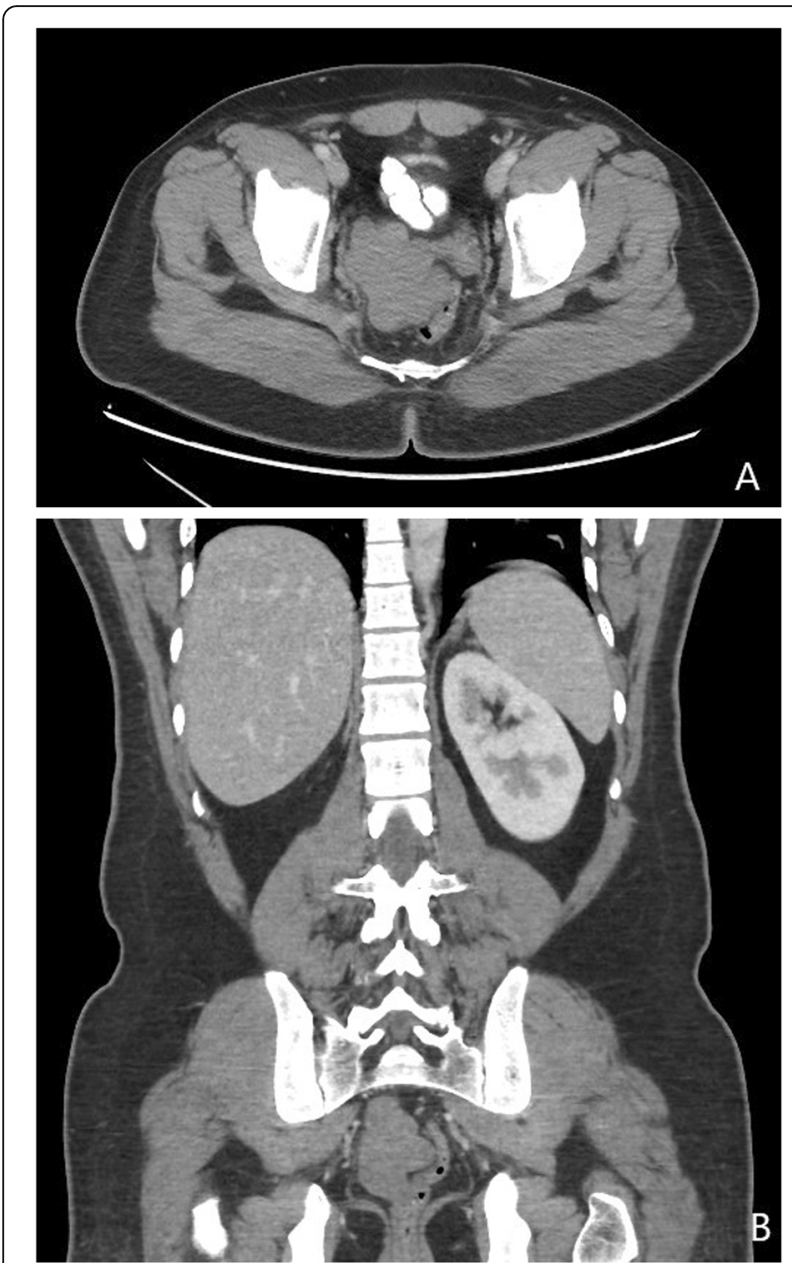

Fig. 2 Post-IV contrast axial (a) and coronal (b) $\subset$ images. The upper abdomen shows single left kidney with absent right kidney. Images in the pelvis showed a lobulated non-enhancing cystic lesion replacing the right seminal vesicle, inseparable from the right upper surface of the prostate and exerting mass effect upon the surrounding structures, upon which MRI was requested

vesicle, the prostate, and the rest of the pelvic structures were seen of normal MRI appearance (size and signal criteria) (Fig. 3).

After discussing the patient's condition and radiological findings with the urology team, the diagnosis of Zinner's syndrome was made that was then discussed with the patient with the different management plans and he preferred that he can live with the current minimal symptoms and decided to go under follow-up after 6 months then yearly by the US and to visit the urological team if he developed any new symptoms.

\section{Discussion}

Seminal vesicle cysts combined with ipsilateral renal agenesis are a rare urological congenital anomaly. Slaoui et al. described that the clinical presentation usually starts between the 2nd and 4th decades of their life
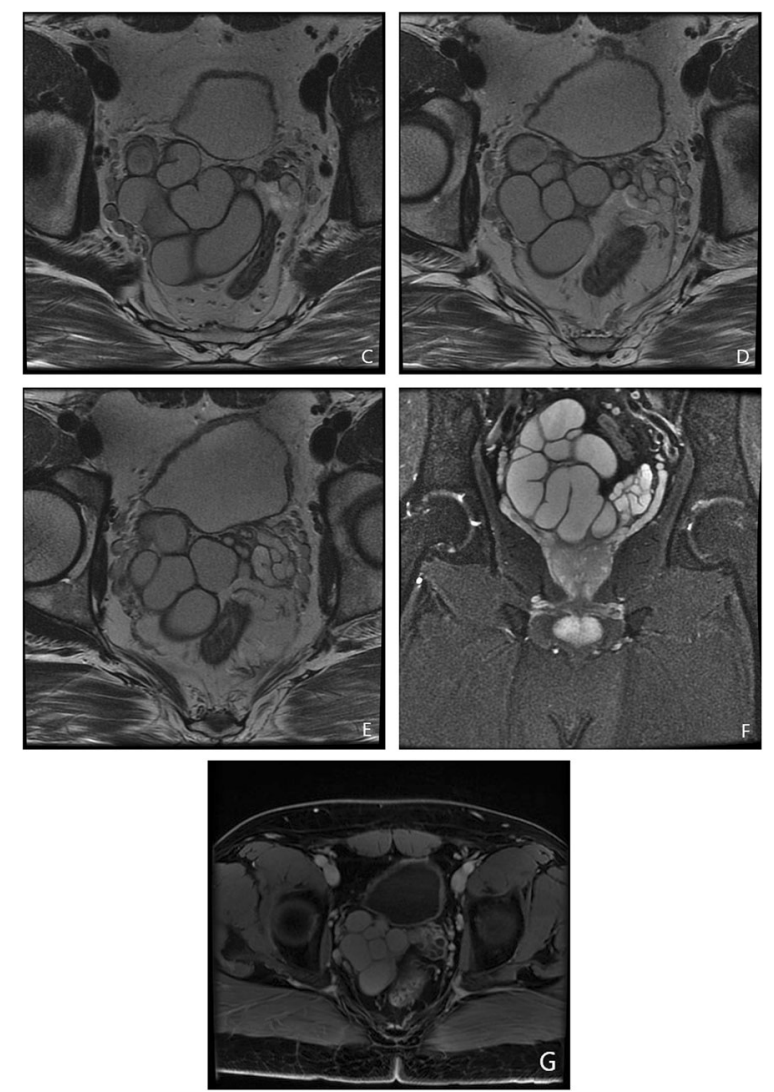

Fig. $3 \mathrm{MRI}$ images - axial T2 WIs (c-e), coronal T2 FS WIs (f), and post-contrast T1 fat sat axial image $(\mathbf{g})$ showing a lobulated tubular fluid-filled cystic lesion replacing the right seminal vesicle with no contrast enhancement in the post-contrast images seen exerting mass effect upon the surrounding structures, while the left vesicle and prostate are seen of normal MRI appearance

caused by the seminal vesicle cysts in the form of bladder irritation, obstruction, and pain mainly in the perineum and scrotum in addition to some reported cases of infertility. Epididymitis is also frequently found as a complication [3]. However, our patient presented with just mild vague abdominal pain and fullness at the pelvis. The diagnostic workup consists of a digital rectal examination, transrectal US, CT scan, and MRI study with or without contrast.

According to Florim et al., the management of this syndrome should be clinically oriented and it can range from follow-up in asymptomatic andminimally symptomatic cases, and that is what was preferred by our patient (otherwise, antibiotics, transurethral aspiration of the seminal vesicle cyst, or transurethral aspiration combined with substance installation (e.g., alcohol and minocycline) is proposed as the conservative treatment), up to invasive treatment that should be considered only in symptomatic patients or patients who failed conservative measurements and that 
usually consists of transurethral resection or balloon dilatation of the ejaculatory duct and open or laparoscopic vesiculectomy [1].

\section{Conclusion}

Although Zinner's syndrome is a rare condition with widely variable clinical presentation and most of the time is non-specific, its unique and characteristic radiological appearance should be enough for the knowledgeable radiologist to be able to give an accurate diagnosis and even sometimes contribute in management.

\section{Abbreviations}

US: Ultrasound; IV: Intravenous; CT: Computed tomography; MRI: Magnetic resonance imaging; CC: Cranio-caudal

\section{Acknowledgements}

No acknowledgements are present.

\section{Authors' contributions}

AS is the main author who gathered the data, wrote the manuscript, and submitted it to the journal. AA contributed to the oversight over the case report and data acquisition. WA contributed to the manuscript preparation. All authors have read and approved the manuscript.

\section{Funding}

No research funding was obtained.

\section{Availability of data and materials}

The datasets used and/or analyzed in the report are available from the corresponding author on reasonable request.

\section{Ethics approval and consent to participate}

The research involving human participants was approved by the ethics committee of the institutions involved (King Khalid Hospital, Najran, Kingdom of Saudia Arabia), and written informed consent was obtained to participate.

\section{Consent for publication}

Written informed consent for publication was obtained from the patient.

\section{Competing interests}

The authors have no conflicts of interest.

Received: 28 July 2020 Accepted: 19 October 2020

Published online: 29 October 2020

\section{References}

1. Florim S, Oliveira V, Rocha D (2018) Zinner syndrome presenting with intermittent scrotal pain in a young man. Radiol Case Reports 13(6):12241227. https://doi.org/10.1016/j.radcr.2018.08.012

2. Juho YC, Wu ST, Tang SH, Cha TL, Meng E (2015) An unexpected clinical feature of Zinner's syndrome - a case report. Urol Case Reports 3(5):149151. https://doi.org/10.1016/j.eucr.2015.06.015

3. Slaoui A, Regragui S, Lasri A et al (2016) Zinner's syndrome: report of two cases and review of the literature. Basic Clin Androl 26(1):1-5. https://doi. org/10.1186/s12610-016-0037-4

\section{Publisher's Note}

Springer Nature remains neutral with regard to jurisdictional claims in published maps and institutional affiliations. 